\title{
Laminar Flow over a Square Cylinder Undergoing Combined Rotational and Transverse Oscillations
}

\author{
B. Anirudh Narayanan ${ }^{1}$, G. Lakshmanan ${ }^{1}$, A. Mohammad ${ }^{2}$ and V. Ratna Kishore ${ }^{1 \dagger}$ \\ ${ }^{1}$ Department of Mechanical Engineering, Amrita School of Engineering, Coimbatore, Amrita Vishwa \\ Vidyapeetham, India \\ ${ }^{2}$ Department of Aerospace Engineering, King Abdulaziz University, Jeddah, Saudi Arabia
}

$\dagger$ Email:v_ratnakishore@cb.amrita.edu

(Received July 2, 2019; accepted June 17, 2020)

\begin{abstract}
This work numerically investigates the effects of combined rotational and transverse oscillations of a square cylinder on the flow field and force coefficients. The primary non-dimensional parameters that were varied are frequency ratio $f_{R}(0.5,0.8), \operatorname{Re}(50-200)$, phase difference $(\phi)$ between the motions and rotational amplitude $\left(\theta_{0}\right)$ with the influence of the last three parameters being discussed in detail. The amplitude of transverse oscillations is fixed at $0.2 \mathrm{D}$, where $\mathrm{D}$ is the cylinder width. The study has been validated using the mean drag coefficient for stationary and transversely oscillating square cylinders from literature. Output data was obtained in the form of force coefficient $\left(\mathrm{C}_{\mathrm{d}}\right)$, vorticity and pressure contours. The governing equations for the 2dimensional model were solved from an inertial frame of reference (overset meshing) using finite volume method. The interplay between the convective field and prescribed motion has been used to explain many of the results obtained. The relative dominance of cylinder motion over the flow stream was determined using Discrete Fast Fourier Transform. The influence of Re on $C_{d}$ disappears when the motions are completely out of phase $(\phi=\pi)$. In general, the $\mathrm{C}_{\mathrm{d}}$ for low Re flows exhibited low sensitivity to change in other parameters. Direct correlation has been observed between frontal area, vortex patterns and drag coefficient
\end{abstract}

Keywords: Bluff body flow; Prescribed motion; Combined oscillations; Vortex shedding; Square cylinder; Two-dimensional laminar flow.

\section{NOMENCLATURE}

A

$\mathrm{Cd}$

$\mathrm{Cl}$

$\mathrm{D}$

f

$\mathrm{f}_{0}$

$f_{R}$

$\mathrm{H} 0$

$\mathrm{L}$

Re

St

$\mathrm{t}$ reference frontal area

coefficient of drag

coefficient of lift

side length of square cylinder

forcing frequency

natural vortex shedding frequency

frequency ratio $\left(\mathrm{f} / \mathrm{f}_{0}\right)$

amplitude of transverse oscillations

length of computational domain

Reynolds Number

Strouhal Number

flow time in seconds
$\mathrm{T} \quad$ time period of oscillation

$\mathrm{U}_{\infty} \quad$ free stream velocity

$\mathrm{v} \quad$ transverse velocity of cylinder

$\alpha \quad$ angle of attack

$\phi \quad$ phase difference

$\theta$ angular displacement due to rotational

oscillation

$\theta_{0} \quad$ rotational oscillation amplitude

$\omega \quad$ angular frequency of oscillation

\section{INTRODUCTION}

The majority of natural and man-made flow scenarios can be categorized as flow over bluff bodies characterized by interesting phenomena such as flow separation and periodic vortex shedding. Cylinders form an important subset of bluff bodies and studies have focused largely on cylinders of circular, square/rectangular and elliptical crosssections. This class of fluid dynamic problems has been meticulously studied over the past century. Canonical bluff body flows have wide ranging applications: FSI problems (Hou et al. 2012), oceanography (Chakrabarti 1994), design of 
buildings (Taniike and Inaoka 1989), bridges (Irwin 2008) and automobiles (Hucho and Sovran 1993), aeronautical and nautical engineering on the macroscale and bio-fluid mechanics, especially heart valve design (Dasi et al. 2009) and MEMS (Ho and Tai 1998) on the micro-scale.

Extensive literature exists for flow over circular and square cylinders, owing to their geometric simplicity and widespread applicability. The early studies by Strouhal (Strouhal 1878), Rayleigh and von Karman laid the foundation for further studies. Roshko (Roshko 1993) provided a detailed review on bluff body flows for circular cylinder and bluff plates over a wide range of $\operatorname{Re}\left(10\right.$ to $\left.10^{7}\right)$, with clear demarcation of different flow regimes (2D,3D, steady and unsteady). Studies have also been conducted to study the effect of prescribed motions (Ongoren and Rockwell 1988) of the cylinder on the flow field.

The existing literature for circular cylinders is humongous. Fixed circular cylinder has been studied (Tritton 1959, Bishop and Hassan 1964, Sarpkaya and Schoaff 1979, Williamson 1996, Silva et al. 2003, Parnaudeau et al. 2008, Rajani et al. 2009) experimentally and numerically. The work by Rajani et al. (2009) in particular establishes the onset of three-dimensionalities in the flow field beyond certain $\mathrm{Re}$ which forms the upper bound of the present study. Later studies shifted focus to oscillating circular cylinders undergoing transverse oscillations (Stansby 1976, Bearman 1984, Williamson and Roshko 1988, Blackburn and Henderson 1999, Celik and Beskok 2009, Kumar and Roy 2016) and rotational oscillations (Thiria et al. 2006, Du and Dalton 2013, Kumar et al. 2013) separately. The combination of rotational and transverse oscillations significantly widens the parameter space and affords better flow control. Blackburn et al. (1999) discovered a propulsive streaming jet regime in quiescent fluid for such combined motion when translation and rotation were completely out of phase $(\Phi=\pi)$, known as the "swimming cylinder". De-constraining the cylinder in the horizontal direction produced a propulsive motion with a terminal velocity of 0.33 times the peak translational velocity. This was further pursued using LBM (Beigzadeh-Abbassi and BeigzadehAbbassi 2012) and in uniform cross flow (Nazarinia et al. 2009, Nazarinia et al. 2012). Koehler et al. (2015) provided a more extended parameter space $(3 \pi / 8 \leq K C \leq 2 \pi, 30 \pi \leq R e \leq 160 \pi, 0 \leq \Phi \leq$ $\pi)$ and identified several new flow regimes broadly classified as horizontal flow, deflected wake, double jet and chaotic regimes. Three-dimensional stability was also investigated

Flow over square cylinders differ considerably from circular cylinders due to the presence of flat faces and sharp corners. An experimental study on fixed square cylinders (Lee 1975) presented pressure fields with special emphasis on onset of turbulence. The experiment was conducted at a fixed $\operatorname{Re}$ of $1.76 \times$ $10^{5}$ and the surface pressure (average and fluctuating), force coefficients (lift and drag) and base pressure were plotted with respect to angle of attack. In an earlier study (Bearman and Trueman
1972), a correlation is provided between wake vorticity strength, distance of vortex shedding and base pressure, which in turn can be related to drag. This correlation finds significant use in the present study. Another experimental study of significance was conducted by Igarashi (1984) in the laminar regime, which studied effects of angle of attack on flow field, especially the leading-edge wake region. The numerical study of laminar flow over fixed square cylinder which set the tone for further work was that by Sohankar et al. (1998). Laminar flows in the range of $45<\operatorname{Re}<200$ and $0<\alpha<45$ was considered. In addition to identifying the $\mathrm{Re}_{\mathrm{cr}}$ for various $\alpha$, the influence of Re and $\alpha$ on drag, lift, St, moment and base suction was numerically determined. Similar work by Lankadasu and Vengadesan (2008) with special focus on the effect of shear was conducted using an inlet of non-uniform velocity. Mat et al. (2011) numerically studied the effect of a splitter plate placed behind the cylinder, similar to the study by Roshko on circular cylinder.

Square cylinders undergoing oscillations have also been studied in detail in the recent years. Drag and lift data, power spectra as well as description of observed phenomena such as lock-in, wake synchronization, vortex switch, etc. were provided at frequencies close to synchronization by Singh et al. (2009) for a transversely oscillating square cylinder at low Re. Another study (Chauhan et al. 2016) experimentally studied flow over a transversely oscillating square cylinder and achieved significant drag reduction with increase in forcing frequency. Other phenomena associated with oscillating square cylinders have also been explored, such as mixing efficiency (Ortega-Casanova 2016), multiple square cylinders (Sewatkar et al. 2011, More et al. 2015, Zhao et al. 2015) and FIV (Joly et al. 2012, Zhao 2015)

The effects of rotation of square cylinders has not been as widely studied (Ohba and Kuroda 1993, Zaki and Gad-El-Hak 1994, De-Ming and Jian-Zhong 2010, Chatterjee and Gupta 2015). Free rotations in cross flow were investigated by Zaki and Gad-ElHak (1994). Ohba and Kuroda (1993) provided force coefficient signals, surface pressure distribution and flow visualizations for rotating square at high Re and low angular velocities. Chatterjee and Gupta (2015) identified suppression of shedding beyond a critical angular velocity along with drag suppression and lift enhancement for a rotating square cylinder. The change in vortex structures when the square is rotated impulsively was studied numerically $(1 \leq$ $R e \leq 300$ ) by De-Ming and Jian-Zhong (2010).

As evident from the literature review, translational and rotational oscillations have been studied separately for square cylinders. The phenomena of lock-in, galloping and better mixing which accompany transversely oscillating square cylinders are of great engineering relevance. Similarly, the occurrence of drag suppression and lift enhancement has been established for rotationally oscillating square cylinders. On the other hand, circular cylinders undergoing combined transverse and rotational oscillations have been observed to produce interesting phenomena such as propulsion (the 
swimming cylinder) and other novel flow regimes (Koehler et al. 2015). However, the effect of this combined motion applied to a square cylinder has not been investigated. The focus of the present work therefore lies in the study of laminar flow over square cylinders undergoing combined transverse and rotational oscillation. This study involves variation of non-dimensional parameters like frequency ratio $f_{R}(0.5,0.8), \operatorname{Re}(50-200)$, phase difference $(\phi)$ between the motions and rotational amplitude $\left(\theta_{0}\right)$.

\section{PROBLEM DESCRIPTION}

The objective of the present work is to study flow over a square cylinder undergoing combined transverse and rotation oscillatory motions. The amplitude of oscillation $\left(\mathrm{H}_{0}\right)$ in the transverse direction is $0.2 \mathrm{D}$, where $\mathrm{D}$ is the cylinder width and the amplitudes $\left(\theta_{0}\right)$ for the rotational oscillation varies from $\pi / 6$ to $\pi / 2$. The frequency of oscillation (f) is the same for both the motions and it is nondimensionalized with the corresponding Strouhal frequency $\left(\mathrm{f}_{0}\right)$ for each Reynolds Number. The Reynolds Numbers ( $\mathrm{Re})$ under consideration are from 50 to 200 . The frequency ratios $f_{R}\left(f / f_{0}\right)$ are 0.5 and 0.8 and the phase difference $(\phi)$ between the two prescribed motions are $\pi / 4, \pi / 2, \pi, 3 \pi / 2$. By varying the above-mentioned parameters, the interaction of the vortices, their formation mechanism and the effect of their interaction on drag coefficient have been studied.

\subsection{Kinematics of Motion}

The square cylinder oscillates transversely with respect to flow, about the $\mathrm{x}$ axis with its centroid always on $x=0$. The rotational oscillation of the cylinder is always about its centroid. As shown in Fig. 1a, at any given instant, $y(t)$ is the displacement of centroid of square cylinder along $y$ axis due to transverse oscillations, measured from $\mathrm{x}$ axis and $\theta(t)$ is the angular displacement of the cylinder due to rotational oscillations, with respect to positive $\mathrm{x}$ axis. The following equations describe the kinematics of the prescribed motion.

$v=H_{\mathrm{o}} \omega \cos (\omega t)$

$\dot{\theta}=\theta_{\mathrm{o}} \omega \cos (\omega t-\phi)$

Where, $\dot{\theta}$ is the angular velocity of the square cylinder at an instant about its centroid.

The waveforms of the two oscillations have been plotted for two extreme cases of phase difference, $\phi=$ $\pi / 2$ and $3 \pi / 2$, in Fig. $1 b$.

\subsection{Computational Domain and Grid}

The computational grid was modelled as two separate grids i.e, foreground mesh and background mesh and these interfaced using an overset interface in ANSYS FLUENT 18. The motion is prescribed to the foreground mesh (represented as (1) in Fig. 2a) which consists of the square, enclosed by a circular mesh of diameter (J) 40D, with the centroid of the square as its center. The far wake length must be sufficiently large enough to avoid distortion of shed vortices due to insufficient wake length. With reference to Koehler, Beran et al. (2015), the downstream wake length was set as 32D. Thus, the background stationary mesh (represented as (2) in Fig. 2a) to which the circular mesh is appended to is a square with width (L) 64D. At any instant of time, the centroid of the background square mesh and the square cylinder always lie on $\mathrm{x}=0$. With respect to the flow domain as illustrated in Fig. 2b, each side of the square cylinder has been spatially discretized into 75 grid elements each (i.e 300 grids in total on the square). Also it can be seen in Fig. 2(b), grid was well resolved near to the wall with first grid distance normal to the wall was kept as D/1000 and growth factor as 1.2. The grid ratio (largest to smallest grid size) was maintained to 3 upto square domain size of 4D.

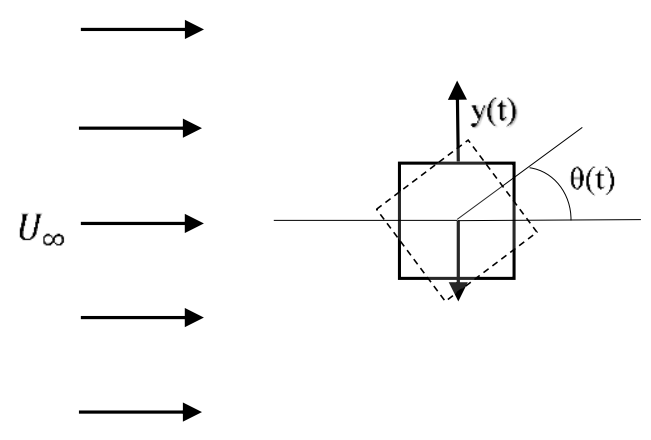

(a)

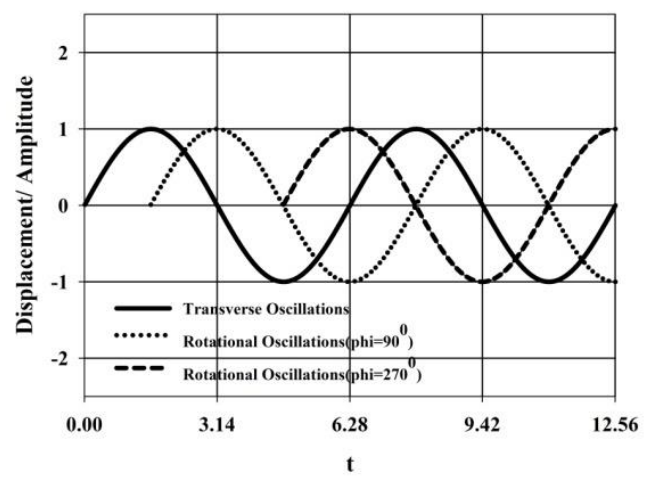

(b)

Fig. 1. (a) General depiction of problem Kinemactics and (b) Waveforms of the transverse and rotational motions.

\section{COMPUTATIONAL METHODOLOGY}

Two-dimensional, unsteady, incompressible NavierStokes equations are solved for flow around the rotating and oscillating square from an inertial frame of reference. COUPLED scheme is used for pressure-velocity coupling. The convective terms in momentum equations are discretized using second order upwind scheme. The temporal discretization is first order. The convergence criteria for momentum 
and continuity equations is set to be $10^{-6}$. The computations were performed using commercial CFD solver, ANSYS FLUENT 18.

As shown in Fig. 2a, at the velocity inlet represented by side eh, $U_{\infty}=$ constant and there is no fluid flow in the y-direction. For the far stream longitudinal boundaries, sides ef and gh, free slip boundary condition is applied. The pressure outlet boundary condition specifying gauge pressure to be zero has been specified at side fg. The surface of the square abcd is a wall, and thus no-slip condition is applied.

The foreground circular mesh zone (1) was moved according to prescribed combined rotational and translational motion using compiled zone motion UDF. The periphery of the foreground circular mesh is the overset interface through which data is interpolated and transferred between the two zones. The working fluid is air.

Since there is a phase difference between the two oscillations, the square cylinder is translated along y direction by a distance from the $\mathrm{x}$ axis corresponding to each phase difference at $t=0$. The free stream velocity for each case based on the parameters of the present study is dependent on the Reynolds number.

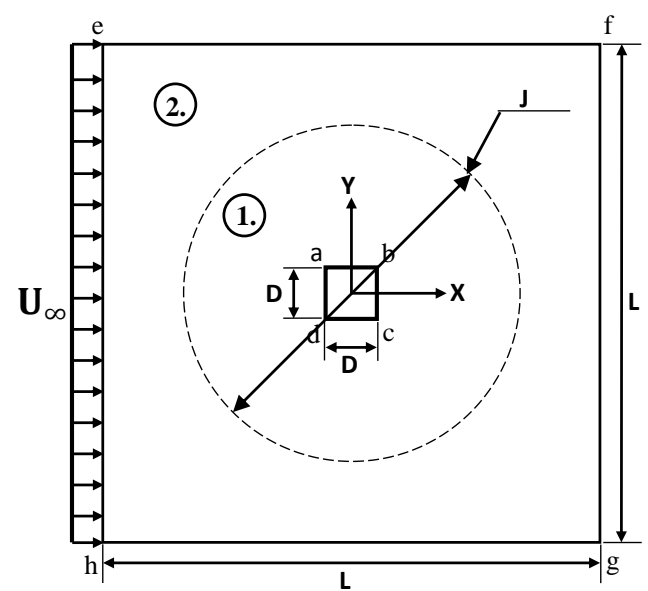

(a)

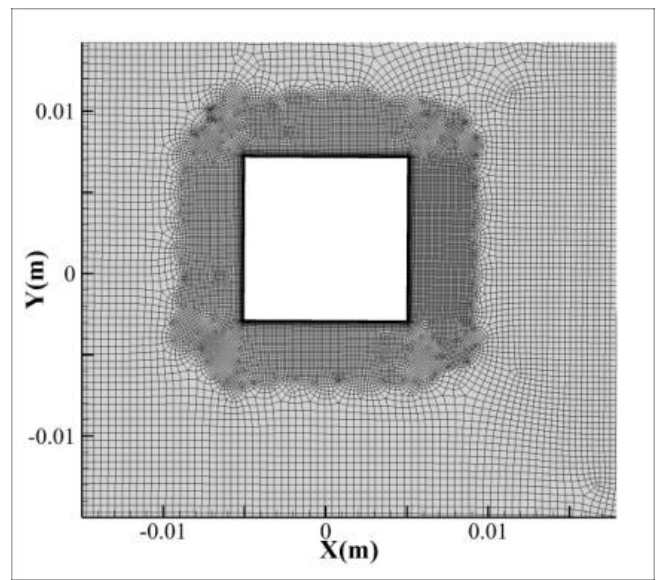

(b)

Fig. 2. (a) Computational Domain (b)Close-up view of mesh around the solid body.

\section{VALIDATION}

\subsection{Stationary Cylinder}

Incompressible flow past a stationary square cylinder for Reynolds numbers 100, 150, 200 was simulated for validating the computational model and the obtained values of Strouhal number (St), average drag coefficient $\left(\bar{C}_{D}\right)$, are compared with the existing data in the literature. Strouhal number is defined as

$S t=f_{0} D / v$

The wake oscillation frequency $\left(\mathrm{f}_{0}\right)$ is calculated from the spectra of the cross-stream velocity $\left(u_{y}\right)$ recorded at a distance of $5 \mathrm{D}$ downstream of the cylinder's rear face along the center-line of the cylinder ( $\mathrm{y}=0)$ as done by Singh et al. (2009). The comparison of $\overline{\mathrm{C}}_{\mathrm{d}}$ and St of present work with data existing in literature is shown in Fig. $3 \mathrm{a}$ and Fig. $3 \mathrm{~b}$ respectively. It can be observed that, both $\overline{\mathrm{C}}_{\mathrm{d}}$ and $\mathrm{St}$ of the present work are well in accordance and within the range of data available in the literature (Sohankar et al. 1998, Darekar and Sherwin 2001, Sahu et al. 2009, Singh et al. 2009, Sen et al. 2011, Ali et al. 2012, Joly et al. 2012, Zhao 2015).

\subsection{Transversely Oscillating Cylinder}

Validation is also done for transversely oscillating square cylinder. Simulations have been run for $f_{R}$ of 0.5 and 1.5 and for $R e=100$. The obtained values of $\overline{\mathrm{C}}_{\mathrm{d}}$ and $\mathrm{C}_{\mathrm{d}, \mathrm{rms}}$ are compared and plotted with that of Singh et al. (2009) in Fig. 3c and 3d respectively. Singh et al. (2009) has obtained a higher value of $\overline{\mathrm{C}}_{\mathrm{d}}$ for flow at $\mathrm{Re}=100$ for a stationary square cylinder compared to other values in the literature as illustrated in Fig. 3c. However, as observed, since both (Singh et al. 2009) and present study have similar profiles i.e. similar slope, it can be concluded that the results obtained for the case of transversely oscillation square cylinder at $\mathrm{Re}=100$ are consistent with that of Singh et al. (2009).

\subsection{Time Step and Grid Independence Test}

Flow past a square cylinder transversely oscillating and rotating at $f_{R}=0.8$, with $\theta_{0}$ and $\phi=90^{\circ}$, at $R e=200$ was simulated for different time increments $(\Delta t)$ to verify the independence of the solution from them. $\overline{\mathrm{C}}_{\mathrm{d}}$ for the different time increments tested is shown in Fig. 4a, where $\mathrm{T}$ is the time period of oscillation for a corresponding frequency. The difference in $\overline{\mathrm{C}}_{\mathrm{d}}$ values for $\mathrm{T} / 400$ and $\mathrm{T} / 800$ increment size is $1.65 \%$ whereas for $\mathrm{T} / 800$ and $\mathrm{T} / 1000$ increment size is $0.002 \%$. Since the change is negligible, $\mathrm{T} / 800$ was chosen as the time increment. To check the effect of grid refinement on coefficient of drag and other desired outputs, computations are done on three grids, coarse grid (G1) having 65230 cells and grid size on square to be D/50, base grid (G2) having 99986 cells in total and grid size on square to be $\mathrm{D} / 75$, finer grid $(\mathrm{G} 2)$ with 306848 cells in total and grid size on square to be $\mathrm{D} / 150$. The average drag coefficient obtained for the refined grid, G3 is 3.263 , for the base grid (G2) is 3.262 and for coarse grid is 3.124. The $C_{d}$ variation with respect to time has been plotted in Fig. 4b. Also, coefficient of lift variation 


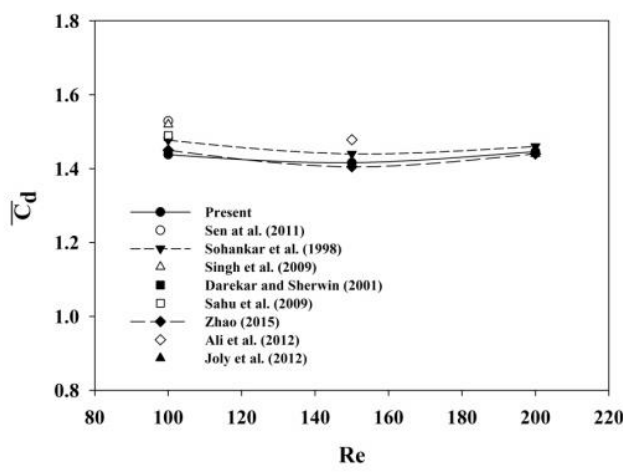

(a)

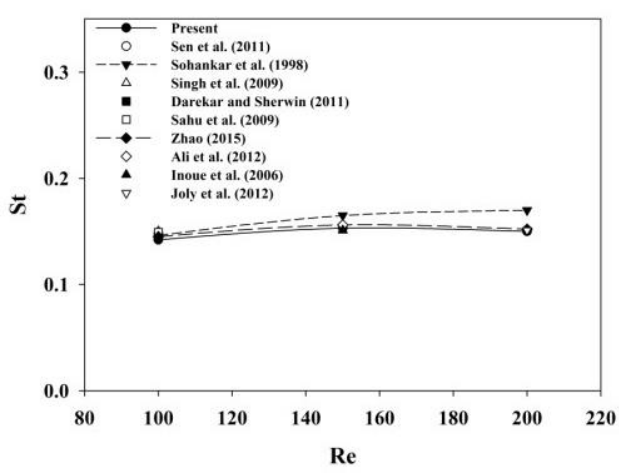

(b)

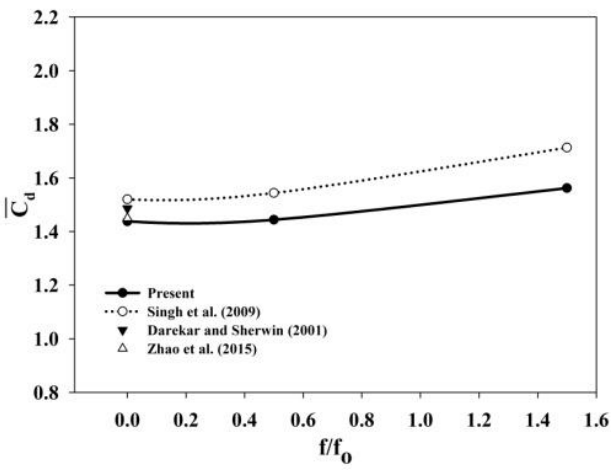

(c)

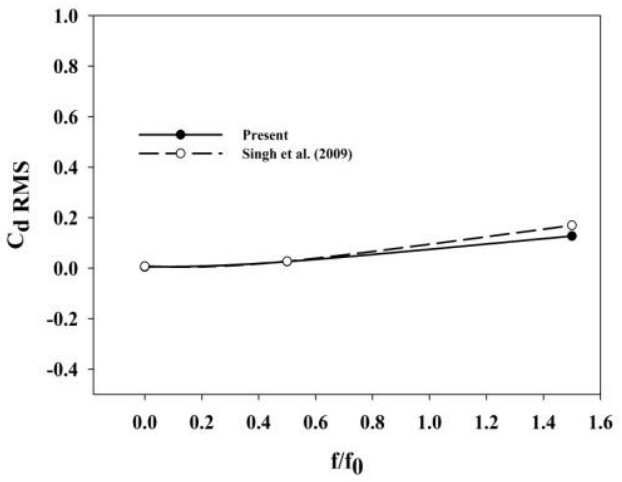

(d)

Fig. 3. Validation curves of stationary square cylinder for (a) mean drag coefficient and (b) Strouhal Number. Validation curves of transversely oscillating square for (c) mean drag coefficient and (d) RMS fluctuating drag coefficient.

with time and snapshot of velocity contours for G2 and G3 were presented in Fig. 4 (c) and (d) respectively. It can be seen from all the flow parameters that solution remains same even when grid in resolved to higher grid density G3, hence all the computations in the present work were simulated using grid $\mathrm{G} 2$.

\subsection{Third Dimensionality Test}

As the Reynolds number increases, the flow becomes $3 \mathrm{D}$ due to instabilities in the third dimension as reported by Rajani et al. (2009). To check for two dimensionality of present study, the extreme case from the parameter domain, i.e., $\mathrm{Re}=200, \mathrm{f}_{\mathrm{R}}=0.8$ with $\theta_{0}$ and $\phi$ of $\pi / 2$ was chosen. For the third dimension in the $3 \mathrm{D}$ mesh, the length of the square cylinder along the z-axis is set at $4 \mathrm{D}$. The spanwise distribution of vorticity magnitude in the $\mathrm{z}$ direction is illustrated in Fig. 4(e). It can be observed that there is no variation in the $\mathrm{z}$ direction, concluding that the flow remains two-dimensional.

\section{RESULTS AND DISCUSSION}

The effect of Reynolds number, phase difference between the two prescribed oscillations and the amplitude of rotational oscillations on the force coefficients is examined in the present work. To avoid the effect of transience of the flow initiation, each case was simulated for at least 20 cycles to obtain asymptotically cyclic results before analyzing them. Chatterjee and Gupta (2015) and Singh et al. (2009) have studied the problems of only rotating and only translating square cylinder respectively. To the best of our knowledge there is no past work that studies drag signals and flow fields for a square undergoing combined rotational and transverse oscillation.

\subsection{Effect of Reynolds Number}

Simulations were done for $\operatorname{Re}=50,100$ and 200, with $\theta_{0}$ and $\phi=90^{\circ}$, to understand the effect and influence of Reynolds Number of the free stream, on the coefficient of drag and the flow features. Fig. 5 shows the variation of drag coefficient with the frequency ratio for different Reynolds Numbers. The $\overline{\mathrm{C}}_{\mathrm{d}}$ value initially decreases and then increases as Re is varied from 50 to 200 . It can be seen that $\bar{C}_{d}$ for $\mathrm{Re}=50$ increases almost linearly as $\mathrm{f}_{\mathrm{R}}$ is increased from 0 to 0.8 . At $\mathrm{Re}=50$ for stationary square cylinder, there is no vortex shedding and instead the wake is a steady recirculation region attached to the bottom and top surfaces of the square. 


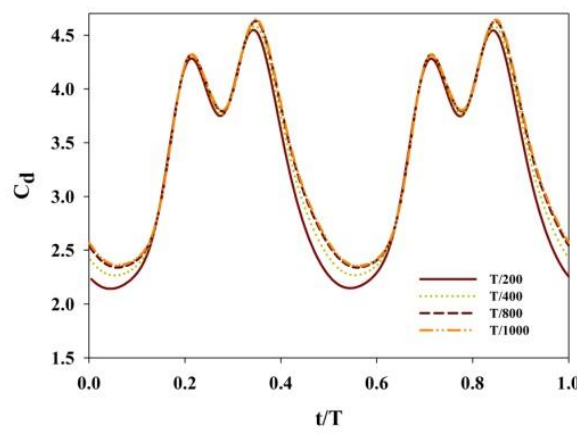

(a)

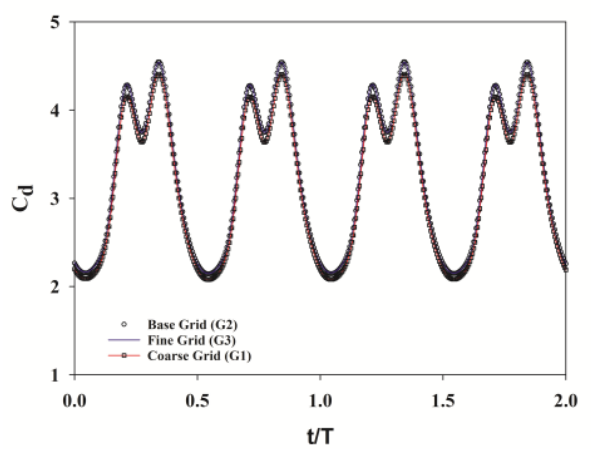

(b)

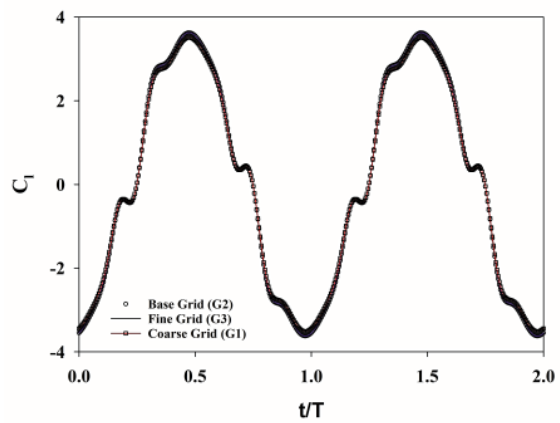

(c)

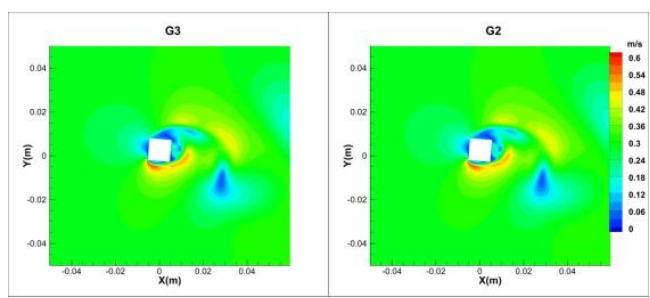

(d)

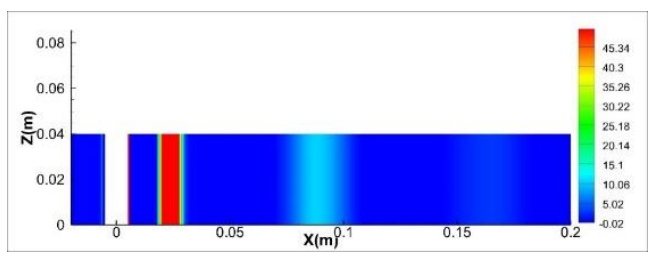

(e)

Fig. 4. (a) Time-step independence and (b) grid independence with $\mathrm{Cd}$ plot (c) grid independence with Cl plot (d) Velocity contours for G2 and G3 grids at $\mathrm{t} / \mathrm{T}=0.05$ (e) Third Dimensionality test for $\operatorname{Re}=200, f_{R}=0.8$.

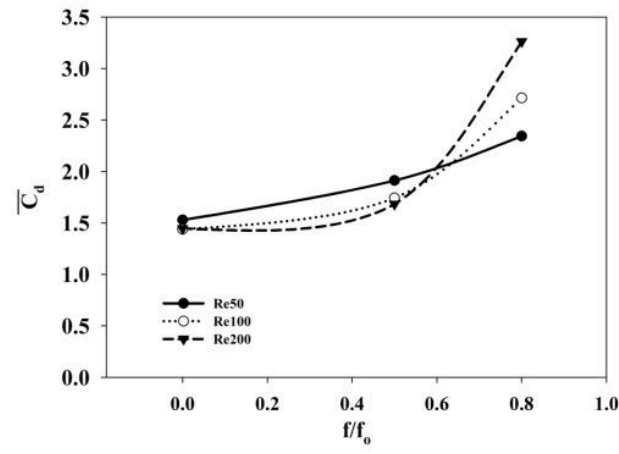

Fig. 5. Variation of mean $C_{d}$ with $f_{R}$.

As the induced oscillation frequency is increased, the wake region destabilises and transitions into vortices that are alternately shed from the top and bottom edges. This results in increased Reynolds stresses in near wake and along with the increase in shear stress on the surface due to rotation, there is an increase in $\overline{\mathrm{C}}_{\mathrm{d}}$. Since the wake region is highly dependent on the prescribed motion, it can be inferred that this linear increase in $\bar{C}_{d}$ for $\mathrm{Re}=50$ is dominated by the induced oscillatory motion compared to the effect of convective field.

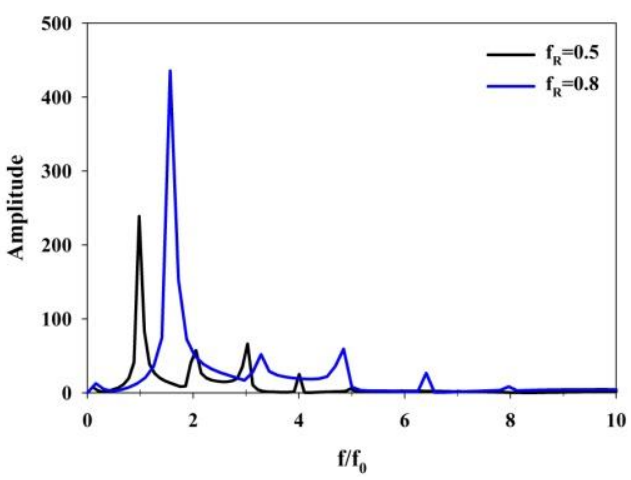

(a)

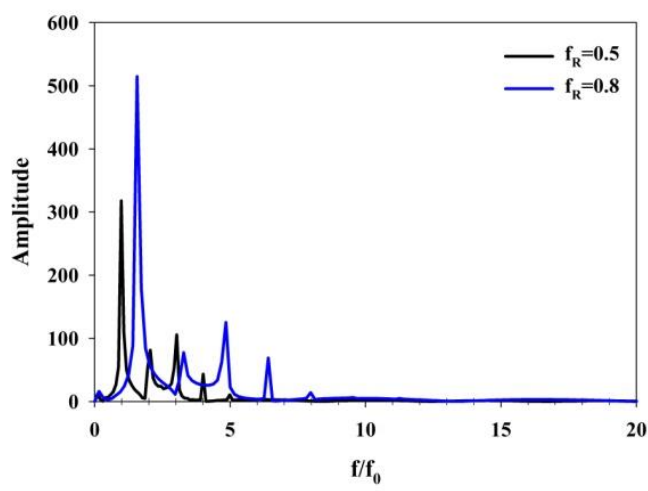

(b)

Fig. 6. FFT Spectrum for (a) $\mathrm{Re}=50$ and (b)

$$
\mathbf{R e}=\mathbf{2 0 0} \text {. }
$$

To further ascertain the dominant frequency in the near wake, spectrum of drag coefficient is obtained by Fast Fourier Transform of the obtained $C_{d}$ signals 


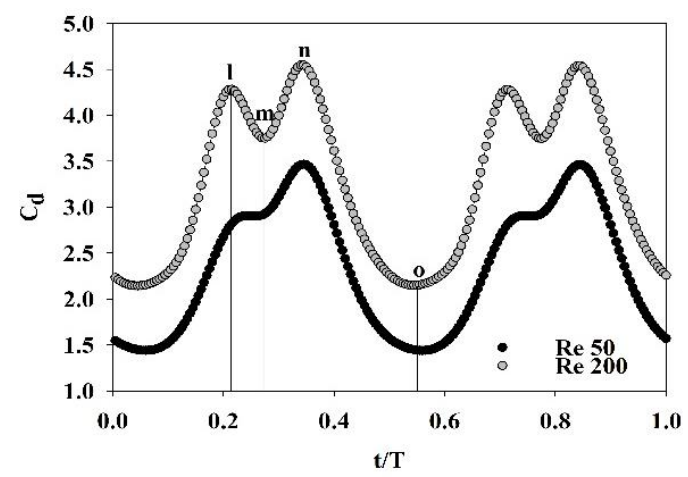

(a)

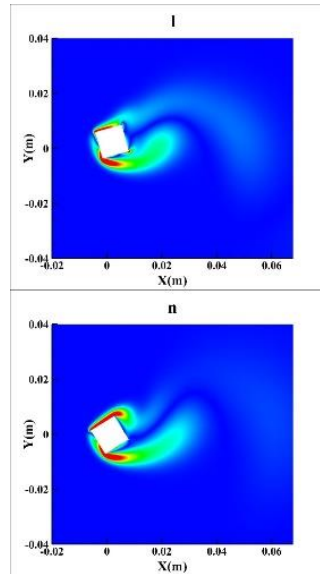

(b)

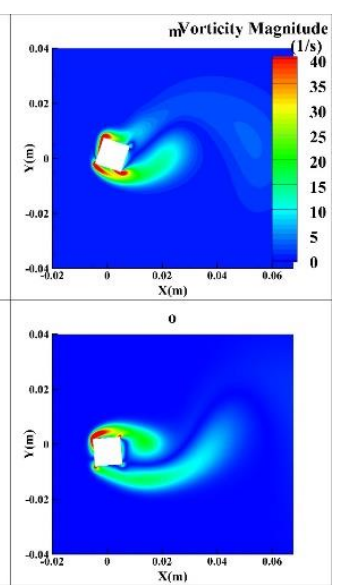

b)

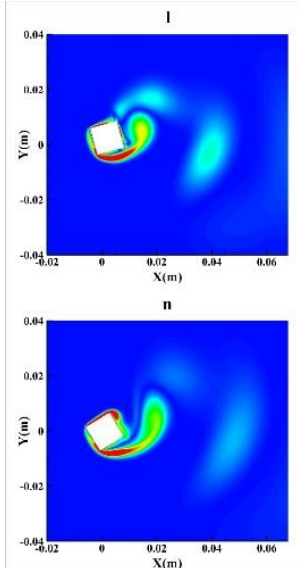

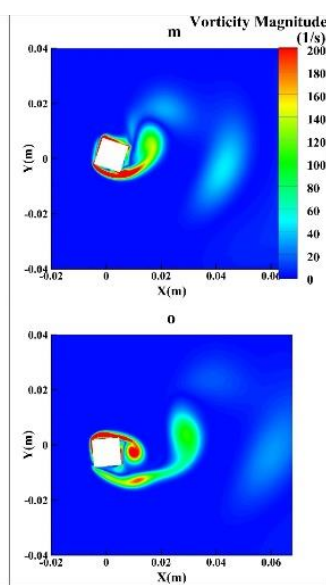

(c)

Fig. 7 (a) Variation of $C_{d}$ with to non-dimensional flow time for $R e=50$ and $200, f_{R}=0.8$. Vorticity magnitude contour plots for $f_{R}=0.8$ at $(b) R e=50$ and $(c) R e=200$. The images $1, m, n$ and $o$ correspond to the non dimensionalized time marked with the corresponding letters in Fig $7 \mathrm{a}$.

for $\mathrm{Re}=50$ and 200 as shown in Fig. 6. The frequency obtained in the $\mathrm{C}_{\mathrm{d}}$ spectra is twice the actual frequency as observed by Singh et al. (2009). In Fig. $6 \mathrm{a}$ and $6 \mathrm{~b}$, it can be observed that peaks are obtained at frequencies corresponding to induced frequency for both $\mathrm{Re}=50$ and 200 respectively. For $\mathrm{f}_{\mathrm{R}}=0.5$, from Fig. $6 a$ and $6 b$, it can be observed that small peaks are also obtained at frequencies corresponding to the natural frequency for both $\operatorname{Re} 50$ and 200 . Since $\mathrm{Re}=50$ has higher value of mean $\mathrm{C}_{\mathrm{d}}$ than $\mathrm{Re}=200$ at $f_{R}=0$, though the increase in $\bar{C}_{d}$ as $f_{R}$ increases from 0 to 0.5 is dominantly influenced by the prescribed motion, due to the discernible effect of the convective flow, Re 50 has higher value of $\bar{C}_{d}$ than $R e=200$ at $f_{R}=0.5$ as seen in Fig. 5. Also, at $f_{R}=0.5$ both the prescribed motion and convective field control the near-wake region. This is similar to the phenomena observed by Singh et al. (2009) outside the lock-in regime for oscillating square cylinder. As the frequency ratio is increased from 0.5 to 0.8 , there is a non-linear increase in $\overline{\mathrm{C}}_{\mathrm{d}}$ for $\mathrm{Re}=200$. This increase in $\bar{C}_{d}$ for $R e=200$ is due to the combined effect of convective flow and prescribed motion. From Fig. 6 , for $\mathrm{Re}=200$, there are much more conspicuous peaks at points corresponding to frequencies other than induced frequency, compared to that of Re 50 arising due to the combined effect of both convective flow and prescribed motion.
To better understand the effect of convective flow field on the drag forces induced on the cylinder, $C_{d}$ with respect to nondimensionalised time for $\mathrm{Re}=50$ and $\mathrm{Re}=200$ have been plotted in Fig. 7a. On observing, $C_{d}$ profile is similar for both $\mathrm{Re}=50$ and 200. Thus, it can be inferred that the mechanism that is initiating the formation of vortices, the interaction of vortices and other factors that affect the drag force on the cylinder are similar for both the Reynolds Numbers. Vorticity magnitude contour plots at the times marked by lines $1, \mathrm{~m}, \mathrm{n}$ and $\mathrm{o}$ on the time scale corresponding to the overlapping inflection points of both the Re as shown in Fig. 7a have been illustrated for both $\mathrm{Re}=50$ and $\mathrm{Re}=200$ in Fig. $7 \mathrm{~b}$ and Fig. 7c respectively.

On comparing Fig. 7b and Fig. 7c, it can be observed that both $\mathrm{Re}=50$ and $\mathrm{Re}=200$ have similar vorticity magnitude contours at the inflection points. This confirms that the mechanism initiating vortex formation in both the cases is same. Thus, it can be concluded only the prescribed motion of the cylinders is initiating the formation of vortices. But it can also be observed that the magnitude of the vortices for $\mathrm{Re}=200$ in Fig. $7 \mathrm{c}$ is much higher compared to the magnitude of vortices for $\mathrm{Re}=50$ in Fig. 7b. When the flow encounters the leading-edge corners of the square, circulation regions are produced which then 
break away from the surface of the cylinder and are carried away into the wake by the flow velocity. When the flow velocity is high, the intensity of the circulation region produced is also high.

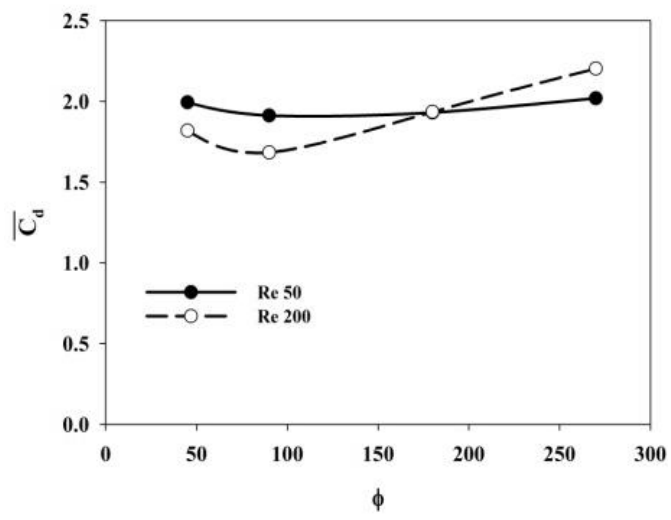

(a)

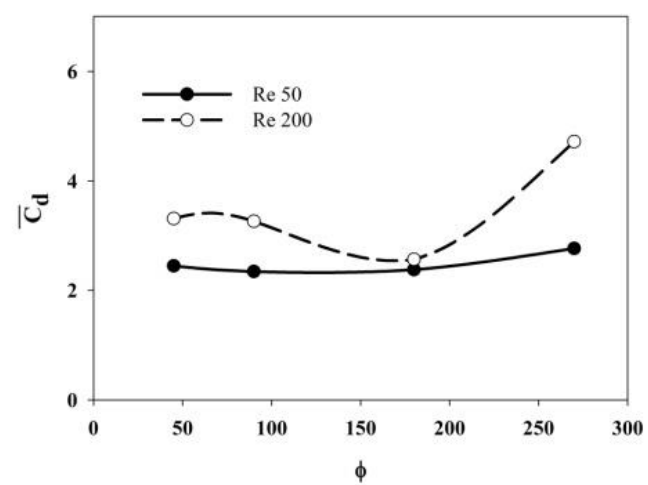

(b)

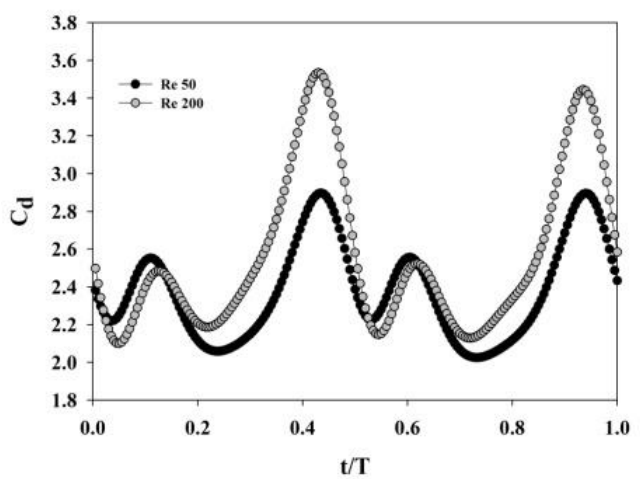

(c)

Fig. 8. Variation of mean $\mathrm{Cd}$ with phase difference for $(a) f_{R}=0.5$ and $(b) f_{R}=0.8$. (c) Variation of Cd with non-dimensional flow time for $\mathrm{Re}=50$ and $200, \mathrm{f}_{\mathrm{R}}=\mathbf{0 . 8}$.

This non-linear increase in $\mathrm{C}_{\mathrm{d}}$ is previously unreported in literature. This is completely opposite to what Chatterjee and Gupta (2015) have observed. For a rotating square cylinder, as $f_{R}$ is increased from 0.5 to $0.8, \mathrm{C}_{\mathrm{d}}$ reduces in value.

\subsection{Effect of Phase Difference}

To understand the effects of the phase difference between the rotational and transverse oscillations on $\overline{\mathrm{C}}_{\mathrm{d}}$ and flow features, Reynolds numbers 50 and $200, \theta_{0}=\pi / 2$ with $\phi$ values of $\pi / 4, \pi / 2, \pi$ and $3 \pi / 2$ are considered. Fig. 8a and Fig. 8b illustrate variation of $\bar{C}_{d}$ for $f_{R}=0.5$ and 0.8. In both Fig. 8a and 8b, there is no significant variation in $\overline{\mathrm{C}}_{\mathrm{d}}$ as $\phi$ is increased from $\pi / 4$ to $\pi / 2$ for both $\mathrm{Re}=50$ and 200 and for $\mathrm{Re}=50$ there is very less variation in the values of $\bar{C}_{d}$ for all the phase differences under consideration. In Fig. $8 \mathrm{~b}$ for $\mathrm{Re}=50$, the values of $\overline{\mathrm{C}}_{\mathrm{d}}$ for $\phi=\pi / 4, \pi / 2, \pi$ and $3 \pi / 2$ are $2.44,2.34,2.37$ and 2.76 respectively with a standard deviation of 0.04 and in Fig. 8a, even for $f_{R}=0.5$, the standard deviation of the values of $\bar{C}_{d}$ for $R e=50$ is 0.16 . Thus, it can be inferred that $\overline{\mathrm{C}}_{\mathrm{d}}$ is not affected by phase difference change for a frequency ratio for $\operatorname{Re}=50$. At $\mathrm{Re}=50$, as stated in the preceding section, there is no vortex shedding happening for a stationary square cylinder. The subsequent wake regimes obtained which can be categorized as "Horizontal streaming to horizontal vortex shedding transition regime", as described by Koehler, Beran et al. (2015), is only due to the increase in oscillation frequency. In both Fig. 8a and Fig. 8b, both $\mathrm{Re}=50$ and 200 have almost the same value of $\bar{C}_{d}$ at $\phi=180^{\circ}$. For further understanding, variation of $C_{d}$ with respect to flow time non-dimensionalized with time period $(\mathrm{T})$ of oscillation has been plotted for a single period in Fig. 8c.

In Fig. 8c, the variation of $\mathrm{C}_{\mathrm{d}}$ for both the Reynolds Numbers, 50 and 200, has similar pattern with different magnitude of troughs and crests. Thus, it can be understood that the dominant mechanism behind the formation of vortices, the interaction of vortices and their resulting effect on $\mathrm{C}_{\mathrm{d}}$ is the same. For further examining, vorticity magnitude contour plots for both $\mathrm{Re}=50$ and $\mathrm{Re}=200$, for $\phi=\pi$ and $\mathrm{f}_{\mathrm{R}}=0.8$ at nondimensionalized time intervals $0.01,0.36,0.49$ and 0.53 represented by images A, B, C and D respectively, have been illustrated in Fig. 9a. In Fig. 9.a.A, vortices are being formed from the corners $\mathrm{w}$ and $\mathrm{z}$. As the square rotates anti-clockwise about its centroid, it can be observed in Fig. 9.a.B, that a circulation region is getting formed around corner $\mathrm{y}$. As the cylinder further rotates, circulation region produced around $\mathrm{y}$ begins to interfere with the circulation region produced by $\mathrm{x}$, as seen in Fig. 9.a.C and 9.a.D. Since the cancelling of leading corner circulation regions by circulation regions around trailing corner as the cylinder rotates happens at both $\mathrm{Re}=50$ and 200, due to the nature of motion (phase difference), there is no effect of $\operatorname{Re}$ at $\phi=\pi$. Drag reduction is a widely studied topic for bluff bodies in fluid flow. For higher Re, this can be seen as a drag suppression mechanism.

The same phenomena is not observed in Koehler, Beran et al. (2015). For a circular cylinder undergoing combined rotational and transverse oscillations, there are no sharp edges that lead to the formation of circulation regions around them. 


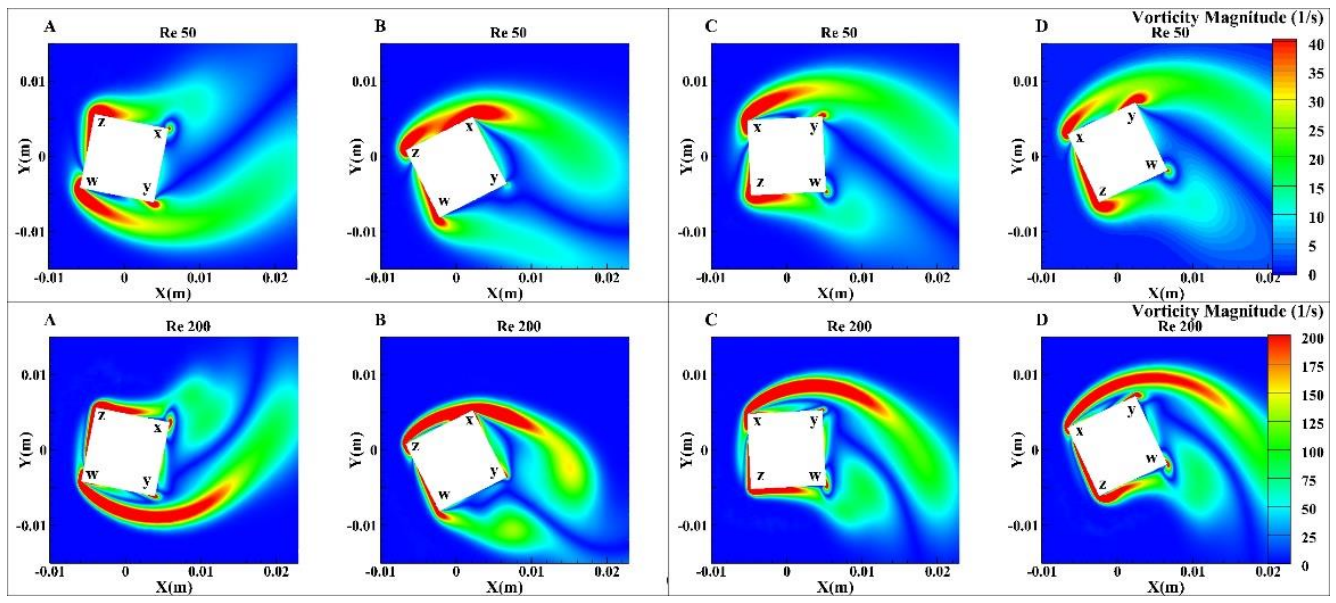

(a)

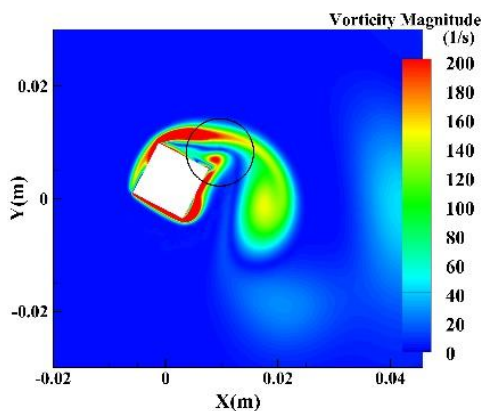

(b)

Fig. 9. Vorticity magnitude contour plots for (a) $\operatorname{Re}=50$ and $\operatorname{Re}=200, \phi=\pi, f_{\mathrm{R}}=0.8, t / T=0.01,0.36$, 0.49 and 0.53. (b) $R e=200, f_{R}=0.8, \phi=3 \pi / 2$.

As seen in Fig. $8 \mathrm{a}$ and 8 b, $\overline{\mathrm{C}}_{\mathrm{d}}$ for $\mathrm{Re}=200$ is higher compared to $\operatorname{Re}=50$, when $\phi=3 \pi / 2$. Vorticity magnitude contour has been plotted for $\mathrm{Re}=200$, $f_{R}=0.8, \phi=3 \pi / 2$ in Fig. 9b. As seen in Fig. 9b, unlike for phase difference $\pi$, here the top leading-edge corner circulation region is taken forward in positive $\mathrm{x}$ direction by flow, thus avoiding it interfering with the circulation region formed around the trailing edge. Since the convective flow complements the effect of the prescribed motion, $\overline{\mathrm{C}}_{\mathrm{d}}$ for $\mathrm{Re}=200$ is higher compared to $\mathrm{Re}=50$ when $\phi=3 \pi / 2$. The abovementioned effect is not seen for $\mathrm{Re}=50$.

\subsection{Effect of Rotational Amplitude}

The rotational amplitude of the square cylinder was varied among $\pi / 6, \pi / 4, \pi / 3$ and $\pi / 2$ for frequency ratios of 0.5 and 0.8 and $\operatorname{Re}$ of 50 and 200 . The phase difference $(\phi)$ between the motions for all the cases was set at $\pi / 2$. The effect of $\theta_{0}$ on the mean $C_{d}$ is plotted in Fig. 10a and 10b. A local minimum is observed at $\theta_{0}=\pi / 4$ for all Re and $f_{R}$, suggesting an underlying drag reduction mechanism. Moreover, for low $\operatorname{Re}(50)$ and $\mathrm{f}_{\mathrm{R}}=0.5$ (Fig. 10a), the variation of $\mathrm{C}_{\mathrm{d}}$ with $\theta_{0}$ is not appreciable $(2.6 \%, 5.9 \%$ and $2.2 \%)$. Also, the mean $C_{D}$ is observed to increase after $\theta_{0}=$ $\pi / 4$, for all $\mathrm{Re}$ and $\mathrm{f}_{\mathrm{R}}$ except for one case $(\mathrm{Re}=200$ and $f_{R}=0.5$ ). Significant drag reduction is observed in this case. The opposite occurs for the same $\operatorname{Re}$ and $\theta_{0}$ at higher $f_{R}$. Considering $R e$ of 200, as $\theta_{0}$ is increased from $\pi / 4$ to $\pi / 2$, the mean $\mathrm{C}_{\mathrm{d}}$ increases by $59.7 \%$ for $f_{R}=0.8$ but drops by $12.3 \%$ for $f_{R}=0.5$ displaying an interesting inversion in trends.

The discussion in this section is based on the following points. First, the generated vorticity is directly related to the surface tangential acceleration, a fact that has been established in literature (Morton 1984).Varying the rotational amplitude, in effect varies the maximum surface velocity and acceleration since the rotational frequency is fixed. Second, the frontal area projected perpendicular to the flow has the strongest influence on the drag value. The minor variations can be deduced from the vorticity field.

Representative tangential acceleration curves of the corner points of the square over one time period have been plotted in Fig. 11. A comparison of magnitudes shows that the transverse amplitude (0.002) is $0.3 \%$ of even the minimum rotational amplitude $(\pi / 6)$. As a result, with increase in rotational amplitude, the effect of superposition subsides, and the effect of rotation dominates the acceleration curve. In fact, Fig. $11 \mathrm{~b}$ is almost a pure sinusoid. This superposition leads to non-uniform distribution of surface acceleration which is the primary surface vorticity production mechanism.

The instantaneous drag coefficient profiles have been plotted at $\mathrm{Re}=200$ for one period (Fig. 12). All profiles display multiple peaks. For instance, $\theta_{0}=\pi / 4$, 
two separated peaks are observed whereas for $\theta_{0}=\pi / 3$, three distinct peaks are seen (Fig. 12c). The profile for $\theta_{0}=\pi / 2$ resembles that of $\pi / 4$ but with two twin peaks.

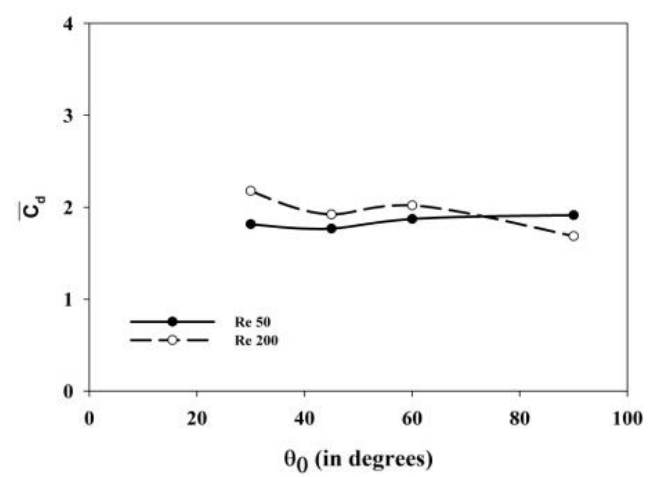

(a)

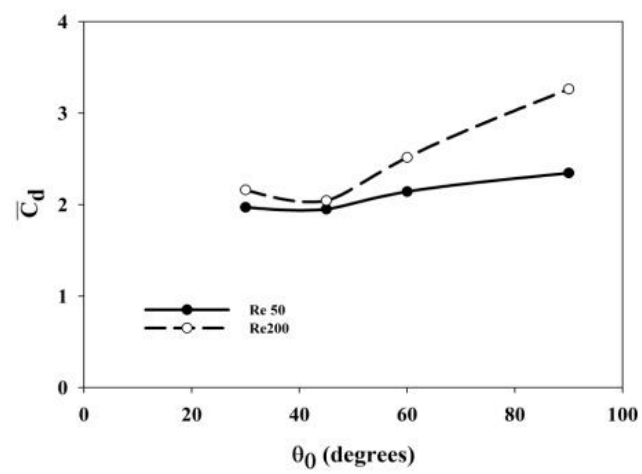

(b)

Fig. 10. Variation of mean $C_{d}$ with $\theta_{0}$ for $f_{R}$ of (a) 0.5 and (b) 0.8 .

The following considerations are necessary for explaining these profiles. It is an established fact that for all $\operatorname{Re}$ (except creeping flow), flow separation for square cylinder always occur at the leading edge (Igarashi 1984). Above a critical Re, this 'leadingedge tripping' induces vortex shedding. Also, since the square is a bluff body, form drag or pressure drag is the major cause of drag. As the cylinder rotates, the projected cross-sectional area normal to the flow varies. The expression for drag coefficient is given by,

$$
\begin{aligned}
\mathrm{C}_{d}=\left(\int\left(\mathrm{p}-\mathrm{p}_{0}\right)(\hat{\mathrm{n}} \cdot \hat{\mathrm{\imath}}) \mathrm{dA}\right) / \rho \mathrm{vA} \mathrm{A}^{2} & \\
& +\left(\int \tau(\hat{\mathrm{t}} \cdot \hat{\mathrm{\imath}}) \mathrm{dA}\right) / \rho \mathrm{vA} \mathrm{A}^{2}
\end{aligned}
$$

The second term on the right can be ignored since viscous drag is relatively negligible for bluff bodies. The reference area $\mathrm{A}$ is the frontal area when the angle of attack is zero. However, the actual frontal area changes with rotation and this significantly affects the drag. The wedge position of the square $(\theta=\pi / 4)$ has the maximum frontal area and corresponds with instances of maximum drag for most cases. The vortices that form behind the cylinder are indications of high drag because they are regions of low pressure. This inverse relationship between vorticity and pressure field helps in correlating the drag to the vortex structure.

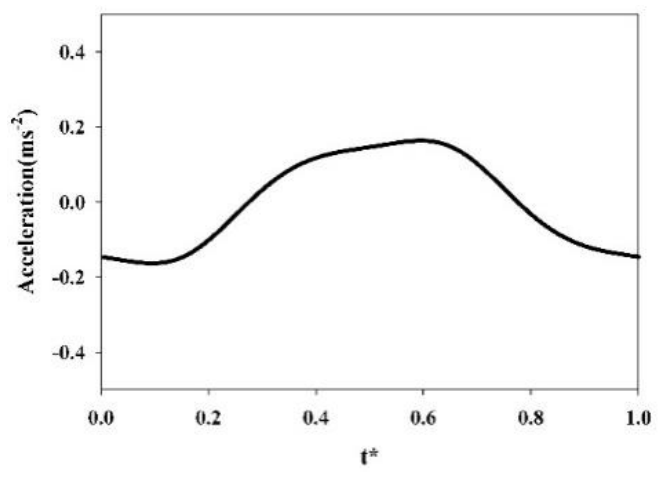

(a)

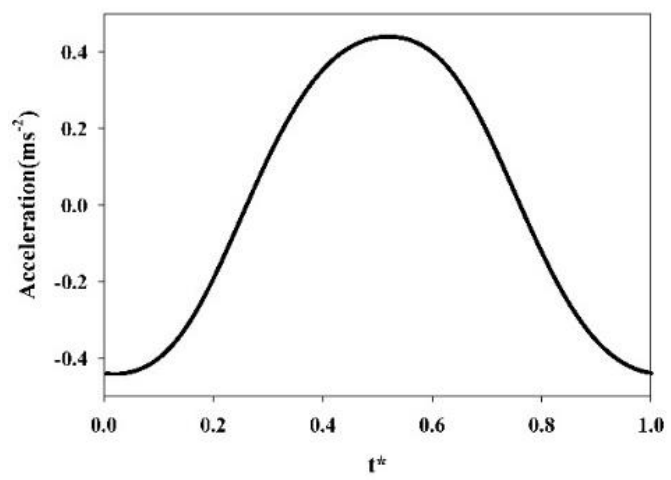

(b)

\section{Fig. 11. Surface tangential acceleration of one corner of the square over a time period for $\operatorname{Re}=200$ and (a) $\theta_{0}=\pi / 6$ and (b) $\theta_{0}=\pi / 2$.}

During a single time period, the wedge position occurs twice, thrice and four times for $\theta_{0}$ of $\pi / 4, \pi / 3$ and $\pi / 2$ respectively. Fig. 13a depicts the vorticity field at for $R e=200$ and $f_{R}=0.5$ at two moments. On comparison with Fig. 12b, the relationship is evident.

Maximum drag occurs when maximum frontal area is projected to the flow, albeit with a minor time lag. Similar relationship is observed for Fig. 13b which resembles a HS regime and Fig. 12d. The twin peaks for $\theta_{0}=\pi / 2$ are caused by the occurrence of wedge form twice during each sense of rotation. The variation observed between different peaks can be understood from the differences in vorticity intensity behind the cylinders. In general, the drag is higher when the vorticity magnitude is higher and when the vortices are in proximity to the cylinder. The same can be observed in Fig. 13b (comparing with Fig. 12d) - Fig. 13b.B and 13b.D have vortices almost on the surface of the cylinder. Moreover, a point of high localized vorticity is seen on the trailing corner for these two instances corresponding with the higher $C_{d}$ peaks among the twin peaks. It is to mention that the high vorticity is not the cause of high drag but a side effect of low pressure, which is the actual cause of both higher drag and vorticity. This relationship can be directly exploited to qualitatively predict drag from vorticity contours. 
B. Anirudh Narayanan et al. / JAFM, Vol. 14, No. 1, pp. 259-273, 2021.

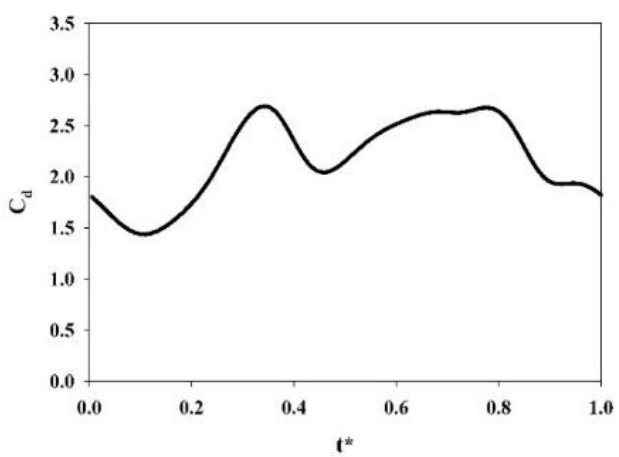

(a) $f_{R}=0.5, \theta_{0}=\pi / 6$

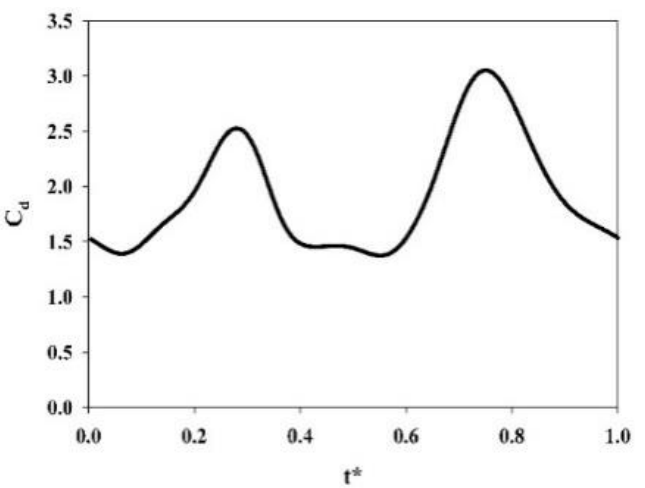

(b) $f_{R}=0.5, \theta_{0}=\pi / 4$

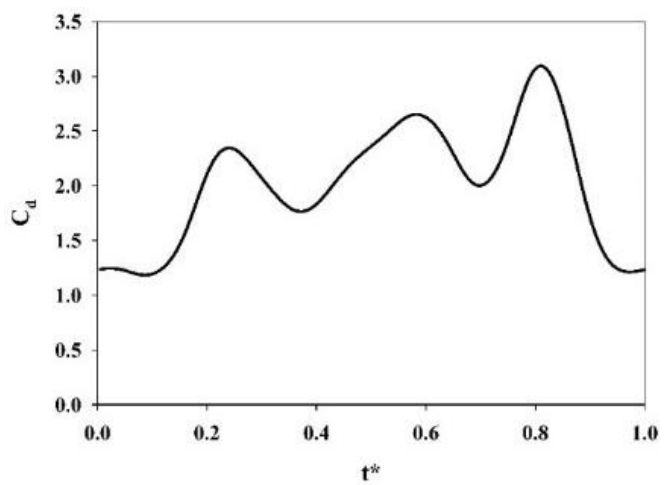

(c) $\mathrm{f}_{\mathrm{R}}=0.5, \theta_{0}=\pi / 3$

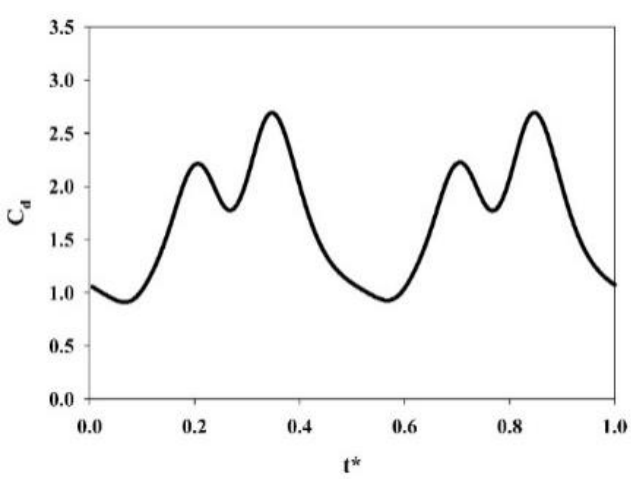

(d) $f_{R}=0.5, \theta_{0}=\pi / 2$

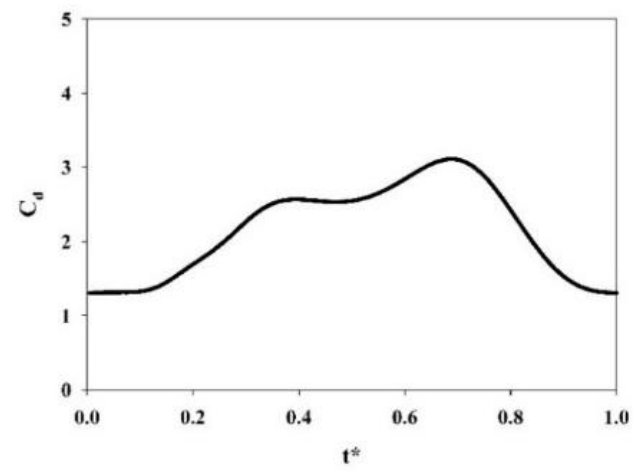

(e) $f_{R}=0.8, \theta_{0}=\pi / 6$

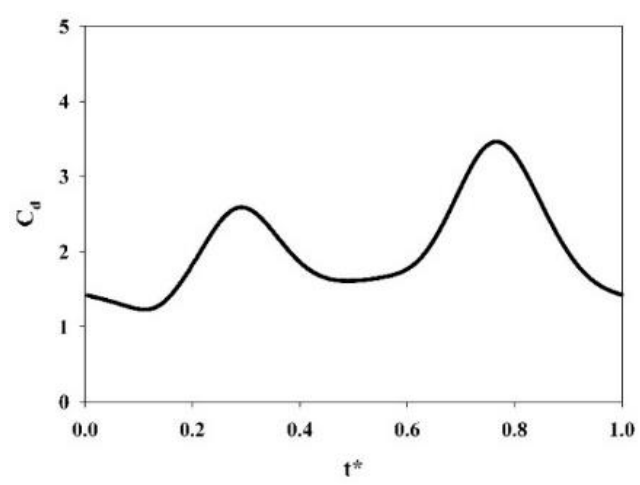

(f) $\mathrm{f}_{\mathrm{R}}=0.8, \theta_{0}=\pi / 4$

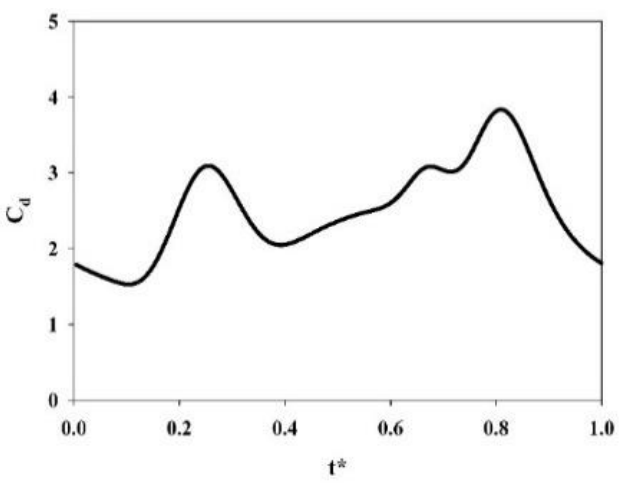

(g) $f_{R}=0.8, \theta_{0}=\pi / 3$

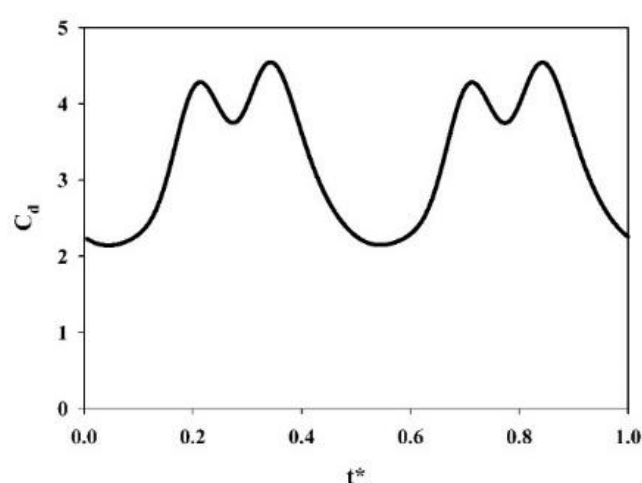

(h) $\mathrm{f}_{\mathrm{R}}=0.8, \theta_{0}=\pi / 2$

Fig. 12. Instantaneous $C_{d}$ profiles for one time period for $\mathbf{R e}=\mathbf{2 0 0}$. 


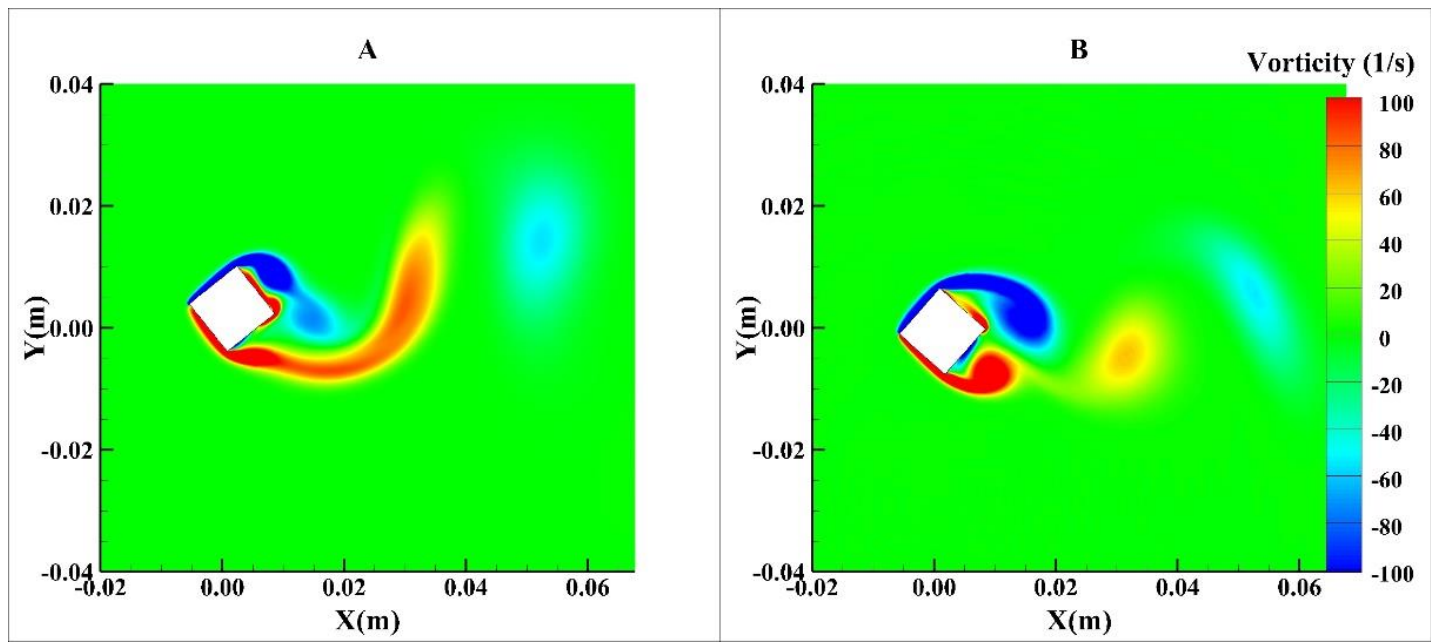

(a)

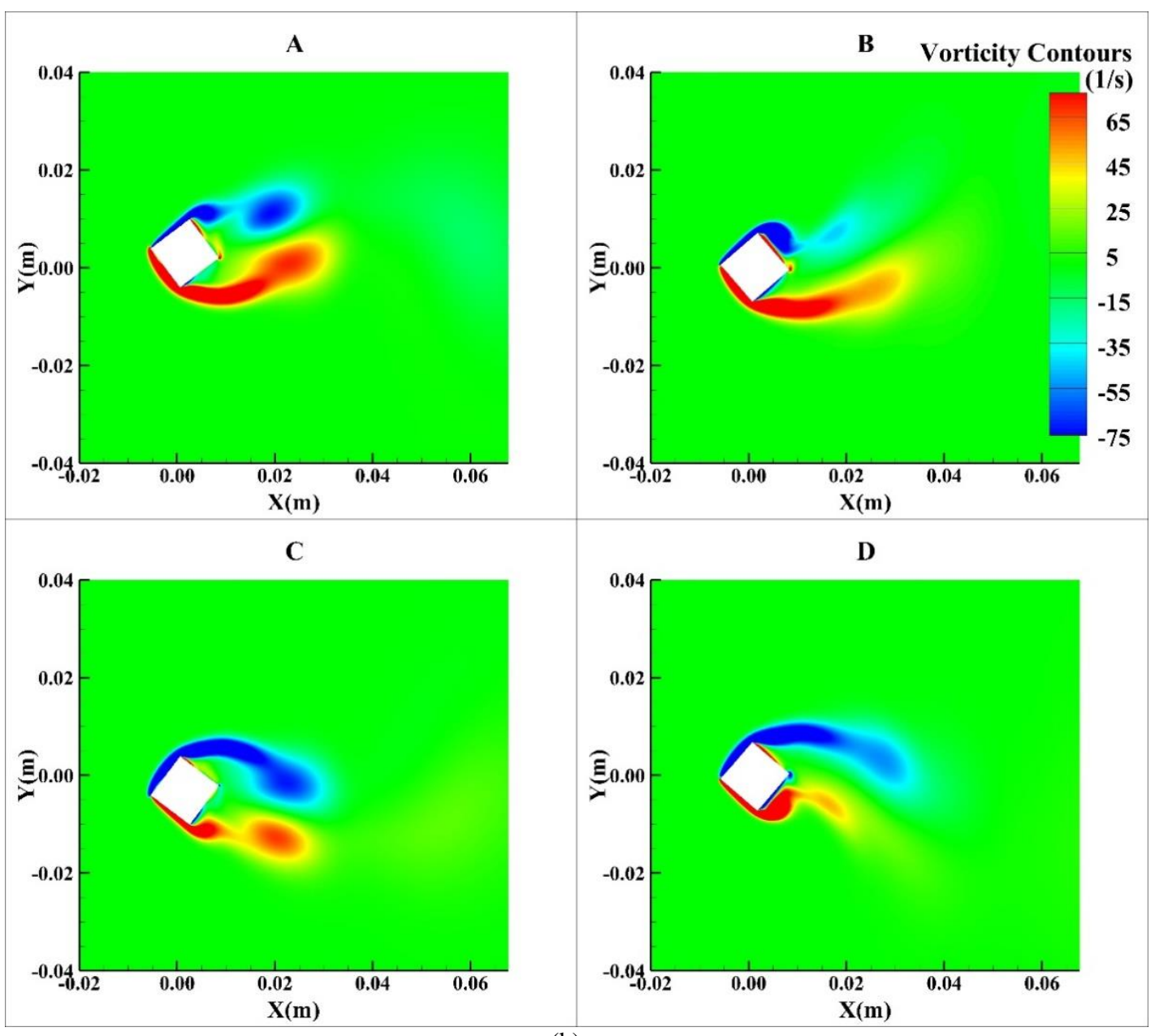

(b)

Fig. 13. (a) Vorticity Contours for $\operatorname{Re}=200, \mathrm{f}_{\mathrm{r}}=0.5$ and $\theta_{0}=\pi / 4$ at $\mathrm{t}^{*}=(\mathrm{A}) 0.26$ and $(\mathrm{B}) 0.75$. (b) Vorticity Contours for $\operatorname{Re}=200, f_{R}=0.5$ and $\theta_{0}=\pi / 2$ at four $t^{*}:(A) 0.17$ (B) 0.33 (C)0.67 and (D)0.83.

\section{Conclusion}

The effect of combined rotational and transverse oscillations of a square cylinder on the flow field and drag are investigated numerically. Four non- dimensional parameters (Reynolds Number, frequency ratio, phase difference, rotational amplitude) are varied individually and the following results were observed.

$\overline{\mathrm{C}}_{\mathrm{d}}$ increases almost linearly with increase in 
oscillation frequency for $\mathrm{Re}=50$ when phase difference and rotational amplitude are constant and this linear increase in $\overline{\mathrm{C}}_{\mathrm{d}}$ is dominated by the induced oscillatory motion compared to the effect of convective field.

There is no significant change in $\overline{\mathrm{C}}_{\mathrm{d}}$ for $\mathrm{Re}=50$, when frequency of oscillation is maintained constant and other parameters are varied.

For $\mathrm{Re}=200$, as the frequency ratio is varied from 0.5 to 0.8 , there is a non-linear increase in $\overline{\mathrm{C}}_{\mathrm{d}}$. This nonlinear increase is because of the combined effect of convective field and prescribed oscillations.

For $\phi=180^{\circ}$, for a particular oscillation frequency, $\overline{\mathrm{C}}_{\mathrm{d}}$ is independent of Reynolds number. Irrespective of $\mathrm{Re}$, the interaction of the vortices is the same due to the nature of prescribed motion (phase difference).

For $\phi=270^{\circ}$, for an oscillation frequency, $\overline{\mathrm{C}}_{\mathrm{d}}$ is always higher for $\mathrm{Re}=200$ as the convective field due to higher flow velocity complements the effect of the prescribed motion.

The $\overline{\mathrm{C}}_{\mathrm{d}}$ exhibits lower sensitivity to $\theta_{0}$ at $\mathrm{Re}=50$ than at $\mathrm{Re}=200$.

The instantaneous drag profiles are directly related to the projected frontal area. The vorticity induced due to cylinder motion plays a secondary role in deciding $\mathrm{C}_{\mathrm{d}}$.

\section{ACKNOWLEDGEMENT}

Apart of computations were performed on the Aziz Supercomputer at King Abdulaziz University's HighPerformance Computing Center (http://hpc.kau.edu.sa/). The authors acknowledge the computer time and technical support provided by the center.

\section{REFERENCES}

Ali, M. S. M., C. J. Doolan and V. Wheatley (2012). Low Reynolds number flow over a square cylinder with a detached flat plate, International Journal of Heat and Fluid Flow 36, 133-141.

Bearman, P. W. (1984). Vortex shedding from oscillating bluff bodies, Annual review of fluid mechanics 16(1), 195-222.

Bearman, P. W. and D. M. Trueman (1972). An investigation of the flow around rectangular cylinders, The Aeronautical Quarterly 23(3), 229-237.

Beigzadeh-Abbassi, M. and M. R. BeigzadehAbbassi (2012). Simulation of self-propulsive phenomenon, using lattice Boltzmann method, Journal of American Science 8(2), 304-309.

Bishop, R. E. D. and A. Y. Hassan (1964). The lift and drag forces on a circular cylinder in a flowing fluid, Proceedings of the Royal Society A 277(1368), 32-50.

Blackburn, H. M., J. R. Elston and J. Sheridan (1999). Bluff-body propulsion produced by combined rotary and translational oscillation, Physics of Fluids 11(1), 4-6.

Blackburn, H. M. and R. D. Henderson (1999). A study of two-dimensional flow past an oscillating cylinder, Journal of Fluid Mechanics $385,255-286$.

Celik, B. and A. Beskok (2009). Mixing induced by a transversely oscillating circular cylinder in a straight channel, Physics of Fluids 21(7), 073601 .

Chakrabarti, S. K. (1994). Fluid structure interaction in offshore engineering (Vol. 1): Computational Mechanics.

Chatterjee, D. and S. K. Gupta (2015). Numerical study of the laminar flow past a rotating square cylinder at low spinning rates, Journal of Fluids Engineering 137(2), 021204.

Chauhan, M. K., S. Dutta, B. K. Gandhi and B. S. More (2016). Experimental Investigation of Flow Over a Transversely Oscillating Square Cylinder at Intermediate Reynolds Number, Journal of Fluids Engineering 138(5), 051105.

Darekar, R. M. and S. J. Sherwin (2001). Flow past a square-section cylinder with a wavy stagnation face, Journal of Fluid Mechanics 426, 263-295.

Dasi, L. P., H. A. Simon, P. Sucosky and A. P. Yoganathan (2009). Fluid mechanics of artificial heart valves, Clinical and experimental pharmacology and physiology 36(2), 225-237.

De-Ming, N. and L. Jian-Zhong (2010). Characteristics of Flow Around an Impulsively Rotating Square Cylinder Via LB-DF/FD Method, Chinese Physics Letters 27(10), 104701.

Du, L. and C. Dalton (2013). LES calculation for uniform flow past a rotationally oscillating cylinder, Journal of Fluids and Structures 42, 40-54.

Ho, C. M. and Y. C. Tai (1998). Micro-electromechanical-systems (MEMS) and fluid flows, Annual Review of Fluid Mechanics 30(1), 579612.

Hou, G., J. Wang and A. Layton (2012). Numerical methods for fluid-structure interaction-a review, Communications in Computational Physics 12(2), 337-377.

Hucho, W. H. and G. Sovran (1993). Aerodynamics of road vehicles, Annual Review of Fluid Mechanics 25(1), 485-537.

Igarashi, T. (1984). Characteristics of the flow around a square prism, Bulletin of JSME 27(231), 18581865 .

Irwin, P. A. (2008). Bluff body aerodynamics in wind engineering, Journal of Wind Engineering and Industrial Aerodynamics 96(6-7), 701-712.

Joly, A., S. Etienne and D. Pelletier (2012). Galloping of square cylinders in cross-flow at low Reynolds numbers, Journal of Fluids and 
Structures 28, 232-243.

Koehler, C., P. Beran, M. Vanella and E. Balaras (2015). Flows produced by the combined oscillatory rotation and translation of a circular cylinder in a quiescent fluid, Journal of Fluid Mechanics 764, 148-170.

Kumar, M. and S. Roy (2016). Immersed boundary method simulation of natural convection over fixed and oscillating cylinders in square enclosure, International Journal of Heat and Fluid Flow 61, 407-424.

Kumar, S., C. Lopez, O. Probst, G. Francisco, D. Askari and Y. Yang (2013). Flow past a rotationally oscillating cylinder, Journal of Fluid Mechanics 735, 307-346.

Lankadasu, A. and S. Vengadesan (2008). Onset of vortex shedding in planar shear flow past a square cylinder, International Journal of Heat and Fluid Flow 29(4), 1054-1059.

Lee, B. E. (1975). The effect of turbulence on the surface pressure field of a square prism, Journal of Fluid Mechanics 69(2), 263-282.

Mat Ali, M. S., C. J. Doolan and V. Wheatley (2011). Low Reynolds number flow over a square cylinder with a splitter plate, Physics of Fluids 23(3), 033602.

More, B. S., S. Dutta, M. K. Chauhan and B. K. Gandhi (2015). Experimental investigation of flow field behind two tandem square cylinders with oscillating upstream cylinder, Experimental Thermal and Fluid Science 68, 339-358.

Morton, B. R. (1984). The generation and decay of vorticity, Geophysical \& Astrophysical Fluid Dynamics 28(3-4), 277-308.

Nazarinia, M., D. L. Jacono, M. C. Thompson and J. Sheridan (2012). Flow over a cylinder subjected to combined translational and rotational oscillations, Journal of Fluids and Structures $32,135-145$.

Nazarinia, M., D. Lo Jacono, M. C. Thompson and J. Sheridan (2009). Flow behind a cylinder forced by a combination of oscillatory translational and rotational motions, Physics of Fluids 21(5), 051701 .

Ohba, H. and S. Kuroda (1993). Numerical analysis of flows around a rotating square cylinder, JSME International Journal Series B Fluids and Thermal Engineering 36(4), 592-597.

Ongoren, A. and D. Rockwell (1988). Flow structure from an oscillating cylinder Part 1. Mechanisms of phase shift and recovery in the near wake, Journal of Fluid Mechanics 191, 197-223.

Ortega-Casanova, J. (2016). Enhancing mixing at a very low Reynolds number by a heaving square cylinder, Journal of Fluids and Structures 65, 120.

Parnaudeau, P., J. Carlier, D. Heitz and E. Lamballais
(2008). Experimental and numerical studies of the flow over a circular cylinder at Reynolds number 3900, Physics of Fluids 20(8), 085101.

Rajani, B. N., A. Kandasamy and S. Majumdar (2009). Numerical simulation of laminar flow past a circular cylinder, Applied Mathematical Modelling 33(3), 1228-1247.

Roshko, A. (1993). Perspectives on bluff body aerodynamics, Journal of Wind Engineering and Industrial Aerodynamics 49(1-3), 79-100.

Sahu, A. K., R. P. Chhabra and V. Eswaran (2009). Two-dimensional unsteady laminar flow of a power law fluid across a square cylinder, Journal of Non-Newtonian Fluid Mechanics 160(2-3), 157-167.

Sarpkaya, T. and R. L. Schoaff (1979). Inviscid model of two-dimensional vortex shedding by a circular cylinder, AIAA Journal 17(11), 11931200.

Sen, S., S. Mittal and G. Biswas (2011). Flow past a square cylinder at low Reynolds numbers, International Journal for Numerical Methods in Fluids 67(9), 1160-1174.

Sewatkar, C. M., A. Sharma and A. Agrawal (2011). Simulation of flow across a row of transversely oscillating square cylinders, Journal of Fluid Mechanics 680, 361-397.

Silva, A. L. F. L. E., A. Silveira-Neto and J. J. R. Damasceno (2003). Numerical simulation of two-dimensional flows over a circular cylinder using the immersed boundary method, Journal of Computational Physics 189(2), 351-370.

Singh, A. P., A. K. De, V. K. Carpenter, V. Eswaran and K. Muralidhar (2009). Flow past a transversely oscillating square cylinder in free stream at low Reynolds numbers, International Journal for Numerical Methods In Fluids 61(6), 658-682.

Sohankar, A., C. Norberg and L. Davidson (1998). Low- Reynolds- number flow around a square cylinder at incidence: study of blockage, onset of vortex shedding and outlet boundary condition, International Journal for Numerical Methods in Fluids 26(1), 39-56.

Stansby, P. K. (1976). The locking-on of vortex shedding due to the cross-stream vibration of circular cylinders in uniform and shear flows, Journal of Fluid Mechanics 74(4), 641-665.

Strouhal, V. (1878). Über eine besondere Art der Tonerregung, nnalen der Physik 241(10), 216251.

Taniike, Y., \& Inaoka, H. (1989). Aeroelastic behavior of tall buildings in wakes. In Advances in Wind Engineering (pp. 317-327): Elsevier.

Thiria, B., S. Goujon-Durand and J. E. Wesfreid (2006). The wake of a cylinder performing rotary oscillations, Journal of Fluid Mechanics 560, 123-147. 
B. Anirudh Narayanan et al. / JAFM, Vol. 14, No. 1, pp. 259-273, 2021.

Tritton, D. J. (1959). Experiments on the flow past a circular cylinder at low Reynolds numbers, Journal of Fluid Mechanics 6(4), 547-567.

Williamson, C. H. K. (1996). Vortex dynamics in the cylinder wake, Annual Review of Fluid Mechanics 28(1), 477-539.

Williamson, C. H. K. and A. Roshko (1988). Vortex formation in the wake of an oscillating cylinder, Journal of fluids and structures 2(4), 355-381.

Zaki, T. G. and M. Gad-El-Hak (1994). Numerical and experimental investigation of flow past a freely rotatable square cylinder, Journal of
Fluids and structures 8(7), 555-582.

Zhao, Guan Meng, Narasimha Rao Pillalamarri, Ravi Chaithanya Mysa, and Rajeev K. Jaiman. "Flow-induced vibrations of single and tandem square columns." In, V002T08A53V02T08A53. American Society of Mechanical Engineers.

Zhao, M. (2015). Flow-induced vibrations of square and rectangular cylinders at low Reynolds number, Fluid Dynamics Research 47(2), 025502. 